\title{
Novel donor-spacer-acceptor compound as the multifunctional component of exciplexes for efficient organic light-emitting diodes
}

\author{
Hao-Yu Yang ${ }^{1,2}$, Cai-Jun Zheng ${ }^{1 *}$, Ming Zhang ${ }^{1,2}$, Jue-Wen Zhao ${ }^{1}$, Yi-Zhong Shi ${ }^{2}$, Chun-Peng Pu ${ }^{1}$, \\ Hui Lin ${ }^{1}$, Si-Lu Tao ${ }^{1}$ and Xiao-Hong Zhang ${ }^{2^{*}}$
}

ABSTRACT By connecting tercarbazole (3Cz) and triphe-
nyltriazine (TRz) units with a diphenyl ether group, we de-
signed and synthesized a new donor-spacer-acceptor structure
molecule 9'-(4-(4-(4,6-diphenyl-1,3,5-triazin-2-yl)phenoxy)
phenyl)-9'H-9,3':6',9"-tercarbazole (3Cz-o-TRz) as the multi-
functional component of exciplex emitters. As expected, the
intermolecular charge-transfer transition is dominant for
3Cz-o-TRz because of the space-enough and conjugation-for-
bidden linkage of the diphenyl ether group, increasing the
intrinsic characteristics of the $3 \mathrm{Cz}$ and TRz moieties in its
single-molecule state. Accordingly, three common electron
acceptors (4,6-bis $(3,5-$ di(pyridin-3-yl)phenyl)-2-methylpyr-
imidine (B3PyMPm), 4,6-bis $(3,5-$ di(pyridin-4-yl)phenyl)-2-
methylpyrimidine (B4PyMPm), and (1,3,5-triazine-2,4,6-triyl)
tris(benzene-3,1-diyl)tris(diphenylphosphineoxide) tris(benzene-3,1-diyl)tris(diphenylphosphineoxide) (PO-
$\mathrm{T} 2 \mathrm{~T})$ ) and three common electron donors $\left(4,4^{\prime}-\right.$ (cyclohexane1,1-diyl)bis( $N, N$-di-p-tolylaniline) (TAPC), tris(4-(10H-phenoxazin-10-yl)phenyl)amine (TPA-PXZ), and N1-phenyl-N4, $N 4$-bis (4-(phenyl( $m$-tolyl)amino) phenyl)-N1-( $m$-tolyl)benzene-1,4-diamine ( $m$-MTDATA)) were chosen to construct six exciplex emitters with $3 \mathrm{Cz}$-o-TRz. In the organic light-emitting diodes (OLEDs), the emission spectra of these exciplex emitters could be gradually modulated from 510 to $590 \mathrm{~nm}$. In addition, TAPC:3Cz-o-TRz- and 3Cz-o-TRz:PO-T2T-based OLEDs achieved desirable performance with maximum external quantum efficiencies (EQEs) around 12\%. Moreover, a simple tandem OLED containing TAPC:3Cz-o-TRz and $3 \mathrm{Cz}-$ $o$-TRz:PO-T2T emitters realized optimal performance with an ultralow turn-on voltage of $2.4 \mathrm{~V}$ and a maximum EQE of $14.1 \%$. These results indicate the great potential of the donorspacer-acceptor structure compounds in developing exciplex emitters.

Keywords: exciplex, donor-spacer-acceptor structure, intermolecular charge-transfer, organic light-emitting diode, multifunctional component

\section{INTRODUCTION}

Since Goushi et al. [1] reported the exciplex-based organic lightemitting diodes (OLEDs) with external quantum efficiencies (EQEs) exceeding the 5\% theoretical limit of conventional fluorescent OLEDs in 2012, exciplex-based OLEDs have aroused great attention [2-9]. The exciplex is well known with an intermolecular charge-transfer (CT) transition between the highest occupied molecular orbital (HOMO) of an electrondonating molecule (D) and the lowest unoccupied molecular orbital (LUMO) of an electron-accepting molecule (A) [10]. With the frontier molecular orbitals (FMOs) located on different molecules, the intermolecular excited states would provide small exchange energies to exciplexes, resulting in extremely small singlet-triplet splittings $\left(\Delta E_{\mathrm{ST}}\right)$. Thus, similar to single-molecule thermally activated delayed fluorescence (TADF) emitters, exciplex emitters can also improve the utilization of triplet excitons through the reverse intersystem crossing (RISC) process and achieve a theoretical internal quantum efficiency of $100 \%$ [11-14]. In addition, the D:A mixed exciplex emitters would naturally have good bipolar electrical conductivity, which optimizes device structures and decreases operating voltages [15-17]. Thus, developing high-performance exciplex emitters is important.

In recent years, a new strategy has been proposed to improve the efficiency of exciplex emitters by introducing bipolar donoracceptor structure compounds as the constituting components [18-20]. In general, donor-acceptor structure molecules can form a CT transition by excitation, which would separate positive and negative charges. Thus, the donor and acceptor moieties in the donor-acceptor structure molecules are expected to possess more spatially separated charges than those without CT formation, which would lead to the formation of exciplexes [19]. To date, the electroluminescence (EL) performance of exciplex emitters has been significantly improved by employing donor-acceptor structure components [21-25]. However, current donor-acceptor structure molecules are purposefully developed for the emitters or hosts of OLEDs. They normally exhibit sole donor-dominated or acceptor-dominated properties because of the strong conjugation between the electron-donating and electron-accepting groups [26-29]. In 2015, Zhang et al. [30] attempted to use the typical donor-acceptor structure compound 10,10'-(sulfonylbis(4,1-phenylene))bis(9,9-dimethyl9,10-dihydroacridine) (DMAC-DPS) to construct exciplex emitters as the D and A components. The maximum EQEs of these exciplex emitters are fairly lopsided in the OLEDs, i.e., 9.1\% with DMAC-DPS as the D component and $1.6 \%$ with DMAC-DPS as the A component. These results indicate that DMAC-DPS is donor-dominated and can only be used as the D

\footnotetext{
${ }^{1}$ School of Optoelectronic Science and Engineering, University of Electronic Science and Technology of China, Chengdu 610054, China

${ }^{2}$ Institute of Functional Nano \& Soft Materials (FUNSOM), Jiangsu Key Laboratory for Carbon-Based Functional Materials \& Devices, Soochow University, Suzhou 215123, China

* Corresponding authors (emails: zhengcaijun@uestc.edu.cn (Zheng CJ); xiaohong_zhang@suda.edu.cn (Zhang XH))
} 
component to construct efficient exciplex emitters. Therefore, current donor-acceptor structure molecules obviously have their inherent limitation. As the components of exciplex emitters, the ideal donor-acceptor structure molecules should simultaneously maintain the intrinsic characteristics of electron-donating and electron-accepting moieties in the single-molecule state.

In this work, we designed and synthesized a new molecule, $9^{\prime}$ (4-(4-(4,6-diphenyl-1,3,5-triazin-2-yl)phenoxy)phenyl)-9' $H$ $9,3^{\prime}: 6^{\prime}, 9^{\prime \prime}$-tercarbazole (3Cz-o-TRz), by using a donor-spaceracceptor structure. In this molecule, the electron-donating group tercarbazole $(3 \mathrm{Cz})$ is connected with the electron-accepting group triphenyltriazine $(\mathrm{TRz})$ through the diphenyl ether group. The former contributes to the separation of FMOs by appending secondary donor groups on a central carbazole to enlarge the delocalization of HOMOs, and the latter is a rigid planar acceptor moiety with a small steric hindrance, which can promote intermolecular interactions. With a space-enough and conjugation-forbidden diphenyl ether linkage, the intramolecular interaction between the $3 \mathrm{Cz}$ and $\mathrm{TRz}$ is efficiently suppressed [31]. Thus, $3 \mathrm{Cz}-\mathrm{O}-\mathrm{TRz}$ is supposed to maintain the intrinsic characteristics of the $3 \mathrm{Cz}$ and $\mathrm{TRz}$ moieties in the single-molecule state and serve as the multifunctional component of exciplex emitters. As expected, the photophysical properties of $3 \mathrm{Cz}-\mathrm{o}$-TRz were mainly characterized by its constituent moieties and the intermolecular CT transition, and $3 \mathrm{Cz}-\mathrm{O}-\mathrm{TRz}$ can be used as the D and A components to construct exciplex emitters. Correspondingly, three common electron acceptors, including 4,6-bis(3,5-di(pyridin-3-yl)phenyl)-2-methylpyrimidine (B3PyMPm), 4,6-bis(3,5-di(pyridin-4-yl)phenyl)-2methylpyrimidine (B4PyMPm), and (1,3,5-triazine-2,4,6-triyl) tris(benzene-3,1-diyl)tris(diphenylphosphineoxide) (PO-T2T), and three common electron donors, including 4,4'-(cyclohexane-1,1-diyl)bis( $N, N$-di- $p$-tolylaniline) (TAPC), $\operatorname{tris}(4-(10 H-$ phenoxazin-10-yl)phenyl)amine (TPA-PXZ), and N1-phenyl$N 4, N 4$-bis (4-(phenyl( $m$-tolyl)amino)phenyl)-N1-( $m$-tolyl)benzene-1,4-diamine ( $m$-MTDATA), were selected to blend with $3 \mathrm{Cz}-\mathrm{o}-\mathrm{TRz}$ to form six exciplex emitters. These exciplexes achieved the modulation of the emission spectra with the peaks varied from 510 to $590 \mathrm{~nm}$. Among them, 3Cz-o-TRz:PO-T2T with $3 \mathrm{Cz}-\mathrm{o}-\mathrm{TRz}$ as the D component and TAPC:3Cz-o-TRz with $3 \mathrm{Cz}-0-\mathrm{TRz}$ as the A component achieved nearly identical performance in the OLEDs with a maximum EQE around $12 \%$. Moreover, a simple tandem OLED simultaneously containing TAPC:3Cz-o-TRz and 3Cz-o-TRz:PO-T2T in the emissive layer (EML) further realized optimal performance with an ultralow turn-on voltage of $2.4 \mathrm{~V}$ and a maximum EQE of $14.1 \%$. These results prove that the donor-spacer-acceptor structure molecules could be an ideal candidate to construct exciplexes as the D or A component and open new possibilities for the structure design of the corresponding OLEDs.

\section{EXPERIMENTAL SECTION}

\section{General methods}

All raw materials and reagents were purchased from commercial suppliers and used without further purification. The ${ }^{1} \mathrm{H}$ and ${ }^{13} \mathrm{C}$ nuclear magnetic resonance (NMR) spectra were recorded by using a Bruker Advance- 400 spectrometer at room temperature. Mass spectra were recorded on a Thermo ISQ mass spectrometer using a direct exposure probe. Density functional theory (DFT) calculation was performed by using the Gaussian 09 program package with the B3LYP/6-31g (d) basis set. Thermogravimetric analysis (TGA) was performed using a TA SDT 2960 instrument under a nitrogen atmosphere. Cyclic voltammetry (CV) was conducted in $\mathrm{N}, \mathrm{N}$-dimethylformamide using a CHI660E electrochemical analyzer. The ultraviolet (UV)-visible absorption spectra were obtained using a Hitachi UV-Vis spectrophotometer U-3900, and the transient photoluminescence (PL) spectra were recorded using a Hitachi fluorescence spectrometer F-4600. PL decays were measured using an Edinburgh Instruments F980 spectrometer.

\section{OLEDs fabrication and characterization}

Indium tin oxide (ITO) glasses with a sheet resistance of $15 \Omega$ per square were used as substrates for device fabrication. Before the device was fabricated, the glasses were first cleaned with acetone, ethanol, and deionized water and then placed in a drying oven at $120^{\circ} \mathrm{C}$ for $2 \mathrm{~h}$. The glasses were treated with UVozone for $30 \mathrm{~min}$ and then placed into a thermal deposition instrument with a high pressure of $5 \times 10^{-4} \mathrm{~Pa}$. All organic layers were deposited onto the glass substrate at a general rate of 1 $2 \AA \mathrm{s}^{-1}$. In general, the deposited rates of $\mathrm{LiF}$ and $\mathrm{Al}$ were controlled at 0.1 and $10 \AA \mathrm{s}^{-1}$, respectively. The optical and electrical data of the devices were respectively recorded with a PR655 Spectrscan and Keithley 2400 Source Meter under ambient atmosphere simultaneously. The current efficiency (CE), power efficiency (PE), and EQE were calculated with the data of current, luminance, and emission, assuming a Lambertian distribution.

\section{RESULTS AND DISCUSSION}

The synthetic scheme of $3 \mathrm{Cz}-\mathrm{o}-\mathrm{TRz}$ is depicted in Scheme 1. First, the electron-donating segment $3 \mathrm{Cz}$ was synthesized in high yields from the Buchwald-Hartwig coupling reaction between carbazole and carbazole derivative, followed by a deprotection reaction with tetrabutylammonium fluoride. Meanwhile, the electron-withdrawing unit 2-(4-bromophenyl)4,6-diphenyl-1,3,5-triazine reacted with the 4-bromophenol to produce TRz-o-PhBr via Williamson reaction. Target compound $3 \mathrm{Cz}-\mathrm{O}-\mathrm{TRz}$ was then synthesized between the $3 \mathrm{Cz}$ and TRz-o$\mathrm{PhBr}$ segments via the Buchwald-Hartwig coupling reaction, and its chemical structure was further confirmed by the NMR and mass spectra (shown in Figs S1-S5).

The thermal properties of $3 \mathrm{Cz}-\mathrm{o}-\mathrm{TRz}$ were measured using TGA and differential scanning calorimetry under a nitrogen atmosphere to evaluate its thermal stability. As shown in Fig. S6, $3 \mathrm{Cz}-\mathrm{o}$-TRz has excellent thermal stability with a decomposition temperature ( $T_{\mathrm{d}}$, corresponding to $5 \%$ weight loss) of $508^{\circ} \mathrm{C}$. Meanwhile, no glass transition was observed in the testing range of 25 to $300^{\circ} \mathrm{C}$. Therefore, $3 \mathrm{Cz}-\mathrm{o}-\mathrm{TRz}$ has enough thermal stability to be used in thermal evaporation.

The complete separation of FMOs is indispensable for the donor-spacer-acceptor structure molecules to serve as multifunctional components of exciplexes. Thus, theoretical calculations were applied on $3 \mathrm{Cz}-\mathrm{o}-\mathrm{TRz}$ to simulate its geometrical structure and molecular orbital distributions. The ground state $\left(\mathrm{S}_{0}\right)$ was optimized by using DFT with a B3LYP/6-31G* basis set. As shown in Fig. 1, the HOMO and LUMO of $3 \mathrm{Cz}-\mathrm{o}$-TRz were completely delocalized on the $3 \mathrm{Cz}$ and $\mathrm{TRz}$ moieties, respectively. Such ideal separated HOMO and LUMO should be attributed to the diphenyl ether spacer, which distinctly suppresses the conjugation between the $3 \mathrm{Cz}$ and $\mathrm{TRz}$ groups. Fur- 

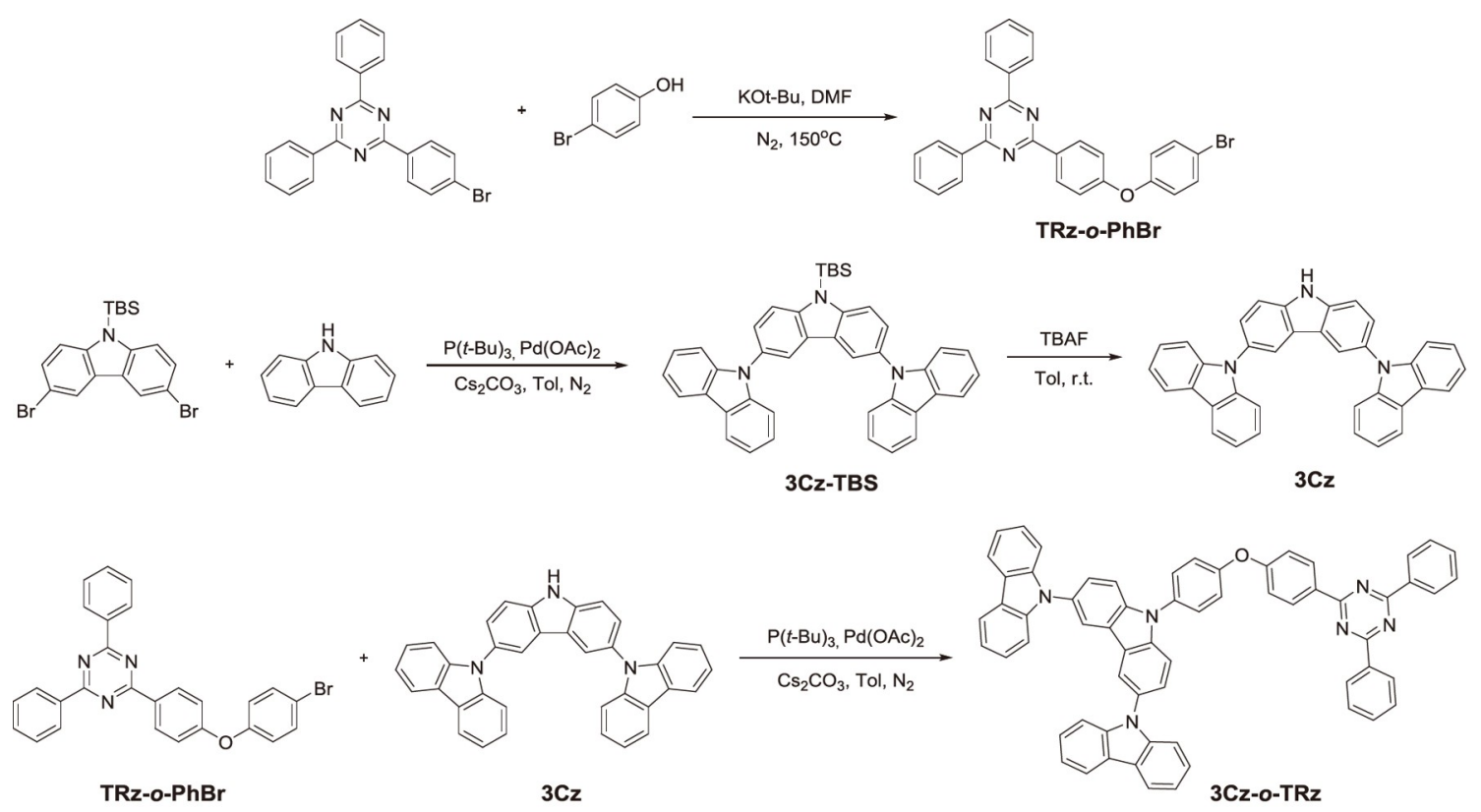

Scheme 1 Synthetic procedure of $3 \mathrm{Cz}-o-\mathrm{TRz}$.
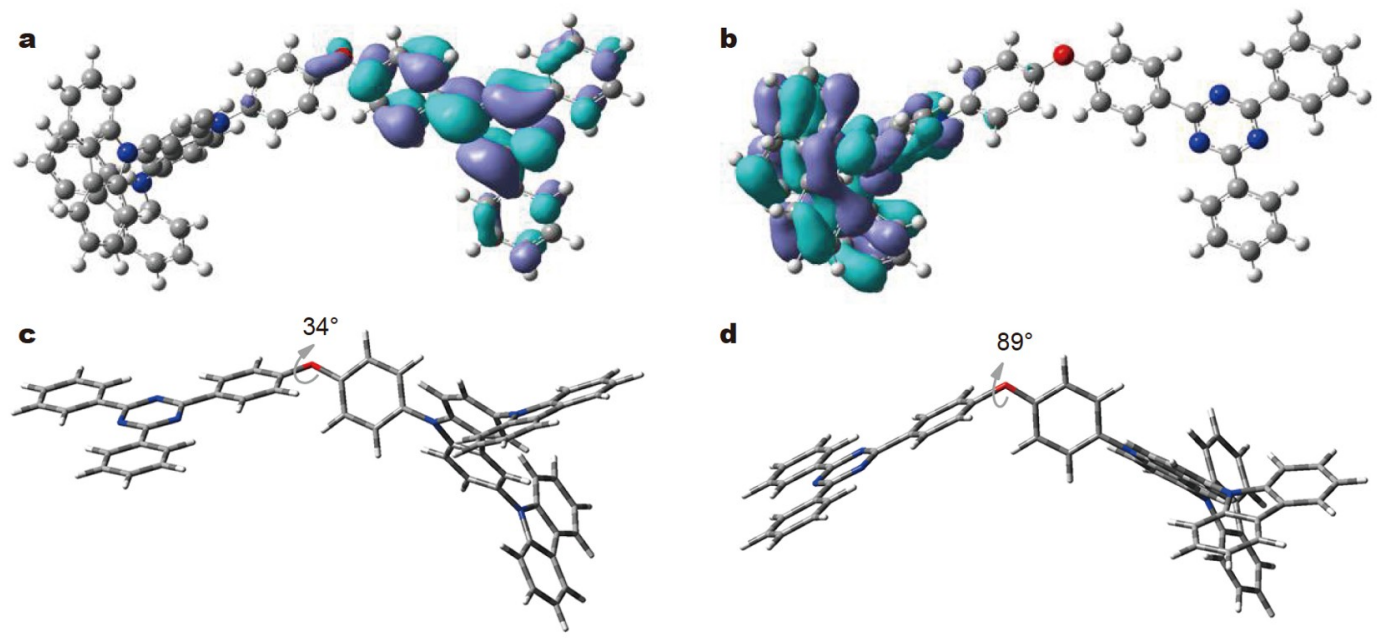

Figure 1 (a) LUMO distributions, (b) HOMO distributions, (c) ground geometry of the $\mathrm{S}_{0}$ state, and (d) the optimized structure of the excited state of $3 \mathrm{Cz}-$ $o$-TRz.

thermore, the centroid distance between the HOMO and LUMO of $3 \mathrm{Cz}-\mathrm{o}-\mathrm{TRz}$ was theoretically estimated at $26.4 \AA$, which is large enough to suppress the spatial CT transition. In specific, the dihedral angle of diphenyl ether changed from $34^{\circ}$ at $\mathrm{S}_{0}$ to $89^{\circ}$ at the adiabatic excited states, indicating that positive and negative charges would be more separated at the excited states. Meanwhile, the oscillator strength of $3 \mathrm{Cz}-\mathrm{O}-\mathrm{TRz}$ was estimated to be only 0.013 , further proving the separation of its FMOs. Relatively isolated donor and acceptor moieties in $3 \mathrm{Cz}-\mathrm{O}-\mathrm{TRz}$ would remarkably increase the intrinsic characteristics from the $3 \mathrm{Cz}$ and $\mathrm{TRz}$ groups in the single-molecule state, ensuring that $3 \mathrm{Cz}-\mathrm{o}-\mathrm{TRz}$ could serve as the D or A component to construct exciplexes.

The photophysical properties of $3 \mathrm{Cz}-\mathrm{o}-\mathrm{TRz}$ and two eigen

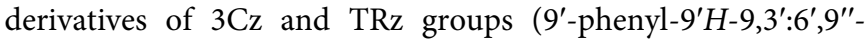
tercarbazole (3Cz-Ph) and 2,4,6-triphenyl-1,3,5-triazine (TriPh-
$\mathrm{TRz})$ ) were investigated to gain an in-depth evaluation of $3 \mathrm{Cz}-\mathrm{O}$ $\mathrm{TRz}$ in the single-molecule state and neat film. Fig. 2a displays the UV-visible absorption and PL spectra of three compounds in dilute toluene solution at room temperature. The absorption of $3 \mathrm{Cz}-\mathrm{o}-\mathrm{TRz}$ (with the maximum absorption at $341 \mathrm{~nm}$ ) is almost composed of those of $3 \mathrm{Cz}-\mathrm{Ph}$ and TriPh-TRz, suggesting that only local excited (LE) transition absorptions exist. Meanwhile, the PL spectrum of $3 \mathrm{Cz}-o-\mathrm{TRz}$ is nearly consistent with that of $3 \mathrm{Cz}-\mathrm{Ph}$. These results indicate that $3 \mathrm{Cz}-\mathrm{O}-\mathrm{TRz}$ only exhibits the intrinsic characteristics of the $3 \mathrm{Cz}$ and $\mathrm{TRz}$ groups in the singlemolecule state. Fig. $2 \mathrm{~b}$ shows the fluorescent spectra of $3 \mathrm{Cz}-\mathrm{O}-$ $\mathrm{TRz}$ in PMMA with different doping concentrations. With the increasing doping concentrations of $3 \mathrm{Cz}-\mathrm{o}$-TRz in PMMA, the relative intensity of LE emission decreased by degrees, whereas a new fluorescence peaked at $445 \mathrm{~nm}$ gradually appeared. As shown in Fig. $2 \mathrm{c}$, the $\mathrm{LE}$ emission in neat $3 \mathrm{Cz}-\mathrm{O}-\mathrm{TRz}$ film 

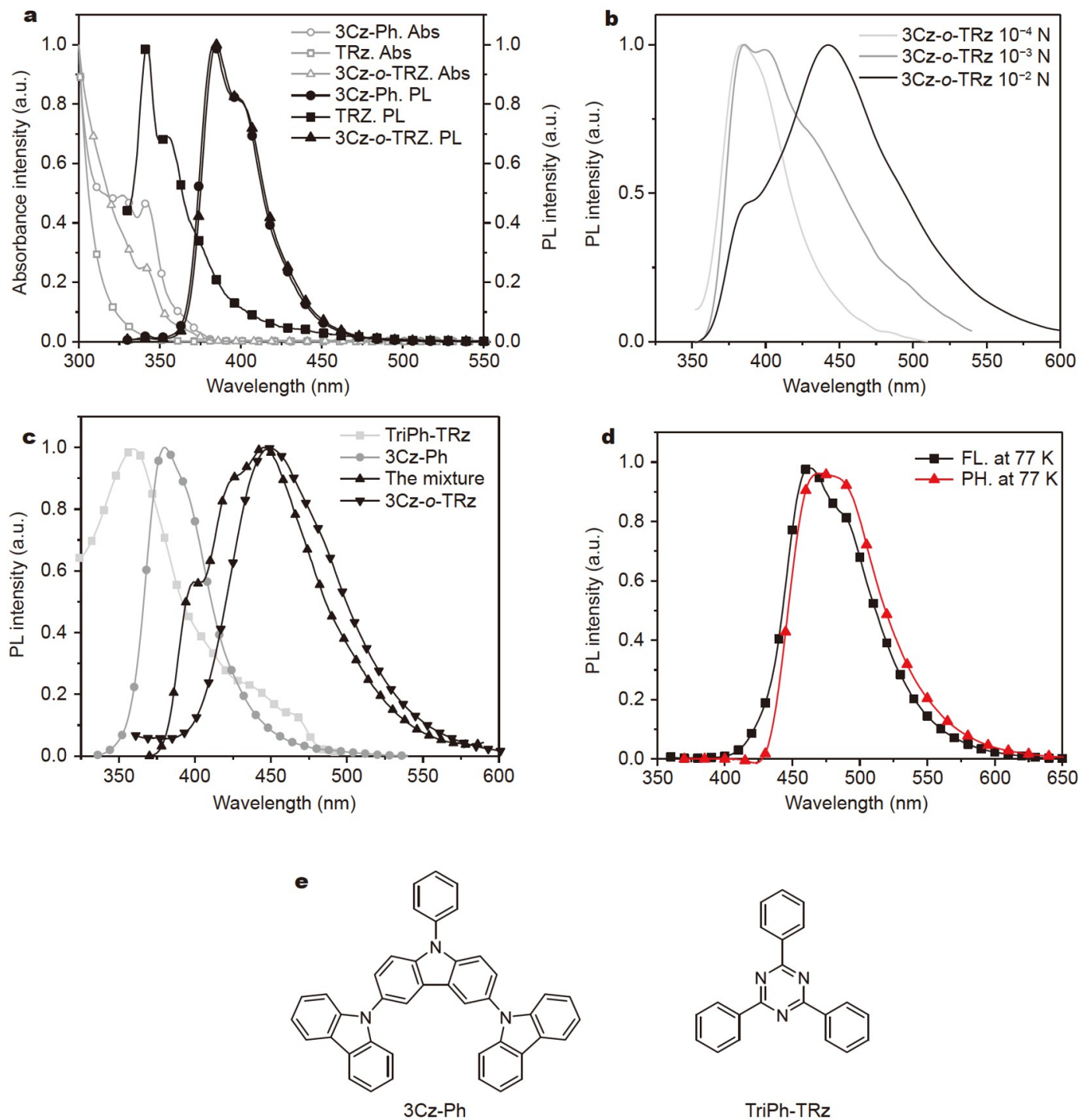

Figure 2 (a) Absorption and PL spectra of $3 \mathrm{Cz}-\mathrm{o}-\mathrm{TRz}, 3 \mathrm{Cz}-\mathrm{Ph}$, and TriPh-TRz in dilute toluene solution; (b) fluorescent spectra of $3 \mathrm{Cz}-\mathrm{o}$-TRz distributed in PMMA with various concentrations; (c) fluorescent spectra of spin-coated films of 3Cz-Ph, TriPh-TRz, 1:1 mixture of 3Cz-Ph and TriPh-TRz and 3Cz-o-TRz; (d) fluorescence and phosphorescence spectra of $3 \mathrm{Cz}-\mathrm{o}$-TRz films at $77 \mathrm{~K}$; (e) structure of eigen derivatives used in the experiment.

completely disappeared, and only a broad and structureless emission peaked at $450 \mathrm{~nm}$, which is consistent with the emission of the 3Cz-Ph:TriPh-TRz blend. Therefore, the new emission could be attributed to the intermolecular CT transition between $3 \mathrm{Cz}-\mathrm{o}-\mathrm{TRz}$ molecules under aggregation state, which further confirms that $3 \mathrm{Cz}-\mathrm{o}-\mathrm{TRz}$ could maintain the intrinsic characteristics of the donor and acceptor moieties. The lowtemperature fluorescence and phosphorescence spectra of $3 \mathrm{Cz}$ $o$-TRz in neat film were then obtained to estimate its lowest singlet $\left(S_{1}\right)$ and triplet $\left(T_{1}\right)$ energy levels under aggregation state. On the basis of the onset positions of the fluorescence and phosphorescence spectra shown in Fig. $2 d$, the $S_{1}$ and $T_{1}$ energy levels were respectively calculated to be 2.88 and $2.84 \mathrm{eV}$. The singlet-triplet splitting $\left(\Delta E_{\mathrm{ST}}\right)$ of $3 \mathrm{Cz}-\mathrm{o}-\mathrm{TRz}$ under aggregation state was accordingly estimated to an extremely small value of $0.04 \mathrm{eV}$, indicating its potential TADF characteristic. In addition, the relatively high $\mathrm{T}_{1}$ level of $2.84 \mathrm{eV}$ would be conducive to the confinement of the excitons on the exciplexes for $3 \mathrm{Cz}-o-\mathrm{TRz}-$ based exciplex emitters [32].
The $\mathrm{CV}$ characterization of $3 \mathrm{Cz}-\mathrm{o}-\mathrm{TRz}$ was performed to evaluate its HOMO and LUMO energy levels. As shown in Fig. S7, from the onsets of the oxidation and reduction curves, the HOMO and LUMO energy levels of $3 \mathrm{Cz}-\mathrm{o}$-TRz were estimated to be -5.86 and $-3.05 \mathrm{eV}$, respectively, which are nearly identical to the results of $3 \mathrm{Cz}$ and $\mathrm{TRz}$ [33]. As shown in Fig. S8, various common $\mathrm{D}$ and $\mathrm{A}$ materials with appropriate FMOs energy levels were selected to construct exciplex emitters with 3Cz-o-TRz.

Fig. 3 displays the PL spectra of the varied blended films based on $3 \mathrm{Cz}-0$-TRz with a ratio of $1: 1$. Among them, the $4,4^{\prime}-N, N^{\prime}-$ dicarbazolylbiphenyl (CBP):3Cz-o-TRz-blended film exhibited the intrinsic emission of $3 \mathrm{Cz}-o-\mathrm{TRz}$, which peaked at $425 \mathrm{~nm}$. This result can be ascribed to the fact that CBP possesses deeper HOMO and shallower LUMO energy levels [17] than $3 \mathrm{Cz}-\mathrm{o}-\mathrm{TRz}$ and no exciplex can form between CBP and 3Cz-o-TRz. By contrast, by mixing $3 \mathrm{Cz}-\mathrm{o}-\mathrm{TRz}$ with three $\mathrm{D}$ materials, $3 \mathrm{Cz}-\mathrm{o}-$ TRz:TAPC, 3Cz-o-TRz:TPA-PXZ, and 3Cz-o-TRz:m-MTDATA exhibited significantly red-shifted PL spectra with peaks at 510 , 

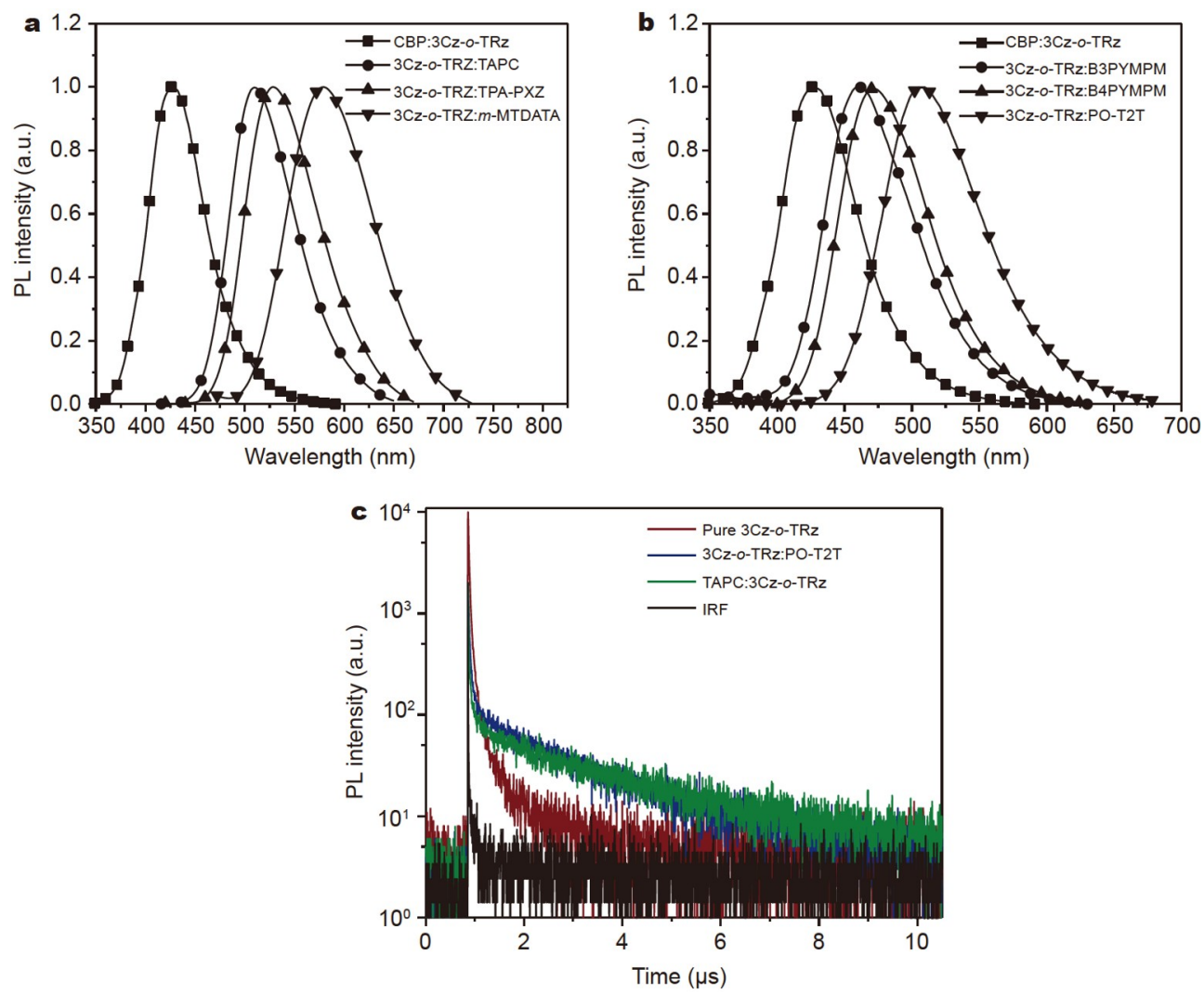

Figure 3 PL spectra of 3Cz-o-TRz-based blend films (1:1) (a) with various D materials and (b) with various A materials; (c) transient fluorescence decays of the 3Cz-o-TRz:PO-T2T, TAPC:3Cz-o-TRz mixed film at $488 \mathrm{~nm}$.

530 , and $580 \mathrm{~nm}$, respectively. Similarly, 3Cz-o-TRz:B3PyMPm, 3Cz-o-TRz:B4PyMPm, and 3Cz-o-TRz:PO-T2T, in which 3Cz$o-\mathrm{TRz}$ was mixed with three A materials, also exhibited redshifted PL spectra peaked at 465,475 , and $510 \mathrm{~nm}$, respectively. These results distinctly indicate that $3 \mathrm{Cz}-\mathrm{o}-\mathrm{TRz}$ could serve as both $\mathrm{D}$ and $\mathrm{A}$ components to construct various exciplexes.

Whether or not these 3Cz-o-TRz-based exciplexes possess effective TADF characteristics was explored. 3Cz-o-TRz:PO-T2T with $3 \mathrm{Cz}-\mathrm{o}-\mathrm{TRz}$ as the $\mathrm{D}$ component and TAPC:3Cz-o-TRz with $3 \mathrm{Cz}-\mathrm{o}-\mathrm{TRz}$ as the A component were selected as the representatives to investigate their transient fluorescence decay. As shown in Fig. 3c and Table S1, the PL decay curves of both exciplexes contained a prompt fluorescence and a delayed fluorescence. In addition, the delayed components of the two exciplexes were evidently stronger than that of pure $3 \mathrm{Cz}-\mathrm{o}-\mathrm{TRz}$, indicating the more efficient RISC process of these exciplexes, which would benefit the exciton utilization in the OLEDs [34]. Accordingly, we further measured the PL quantum yields (PLQYs) of 3Cz-o-TRz:PO-T2T and TAPC:3Cz-o-TRz under air atmosphere. Their absolute PLQYs were 68\% for TAPC:3Cz-oTRz and $66 \%$ for 3Cz-o-TRz:PO-T2T.

Subsequently, the EL performance of these six 3Cz-o-TRzbased exciplex emitters was evaluated. The OLEDs based on the exciplex emitters with $3 \mathrm{Cz}-o-\mathrm{TRz}$ as the A component were fabricated with a structure of ITO/TAPC (or TPA-PXZ or $m$ MTDATA) $(40 \mathrm{~nm}$ )/TAPC (or TPA-PXZ or $m$-MTDATA):3Cz$o$-TRz $(20 \mathrm{~nm}) / \mathrm{TmPyPB}(50 \mathrm{~nm}) / \mathrm{LiF}(1 \mathrm{~nm}) / \mathrm{Al}$. Among them, ITO and LiF/Al worked as the anode and the cathode, respectively; the corresponding D material and 3,3'-(5'-(3-(pyridin-3- yl)phenyl)-[1,1':3',1"-terphenyl]-3,3"-diyl)dipyridine (TmPyPB) were respectively used as the hole-transporting layer (HTL) and the electron-transporting layer (ETL). Meanwhile, the OLEDs based on the exciplex emitters with $3 \mathrm{Cz}-o$-TRz as the $\mathrm{D}$ component were fabricated with a structure of ITO/TAPC $(30 \mathrm{~nm}) /$ $3 \mathrm{Cz}-\mathrm{o}-\mathrm{TRz}(10 \mathrm{~nm}) / 3 \mathrm{Cz}-\mathrm{o}$-TRz:B3PyMPM (or B4PyMPM or PO-T2T) $(20 \mathrm{~nm}) / \mathrm{B} 3$ PyMPM (or B4PyMPM or PO-T2T) $(50 \mathrm{~nm}) / \mathrm{LiF}(1 \mathrm{~nm}) / \mathrm{Al}$. In particular, a thin layer of $3 \mathrm{Cz}-o-\mathrm{TRz}$ was introduced into the device to prevent the formation of exciplex between the HTL and the EML.

The key performances of these OLEDs are illustrated in Fig. 4 and listed in Table 1. The EL spectra of six exciplex emitters all red-shifted relative to their PL spectra, which peaked at 524, 540, and $588 \mathrm{~nm}$ for TAPC:3Cz-o-TRz, TPA-PXZ:3Cz-o-TRz, and $m$ MTDATA:3Cz-o-TRz and at 516, 520, and $520 \mathrm{~nm}$ for $3 \mathrm{Cz}-\mathrm{o}-$ TRz:B3PyMPm, 3Cz-o-TRz:B4PyMPm, and 3Cz-o-TRz:PO$\mathrm{T} 2 \mathrm{~T}$, respectively. Meanwhile, all the six devices exhibited relatively low turn-on voltages (at the brightness of $1 \mathrm{~cd} \mathrm{~m}^{-2}$ ) around $2.6 \mathrm{~V}$ because of the good carrier injection and transport capabilities of exciplex emitters. Among the six exciplex emitters, $m$-MTDATA:3Cz-o-TRz exhibited the lowest efficiencies in the device. This result can be ascribed to the fact that the narrow bandgap of orange-red exciplex emitter greatly increases the non-radiative decays of excited states [35]. By contrast, the green OLEDs with TAPC:3Cz-o-TRz and 3Cz-o-TRz:PO-T2T as emitters achieved maximum CEs and EQEs of 39.3 and $37.2 \mathrm{~cd} \mathrm{~A}^{-1}$ and $12.1 \%$ and $11.8 \%$, respectively, and remained at high values of $11.5 \%$ and $11.0 \%$ at a luminance of $1000 \mathrm{~cd} \mathrm{~m}^{-2}$. These results are consistent with their PLQY results. With $3 \mathrm{Cz}-$ 

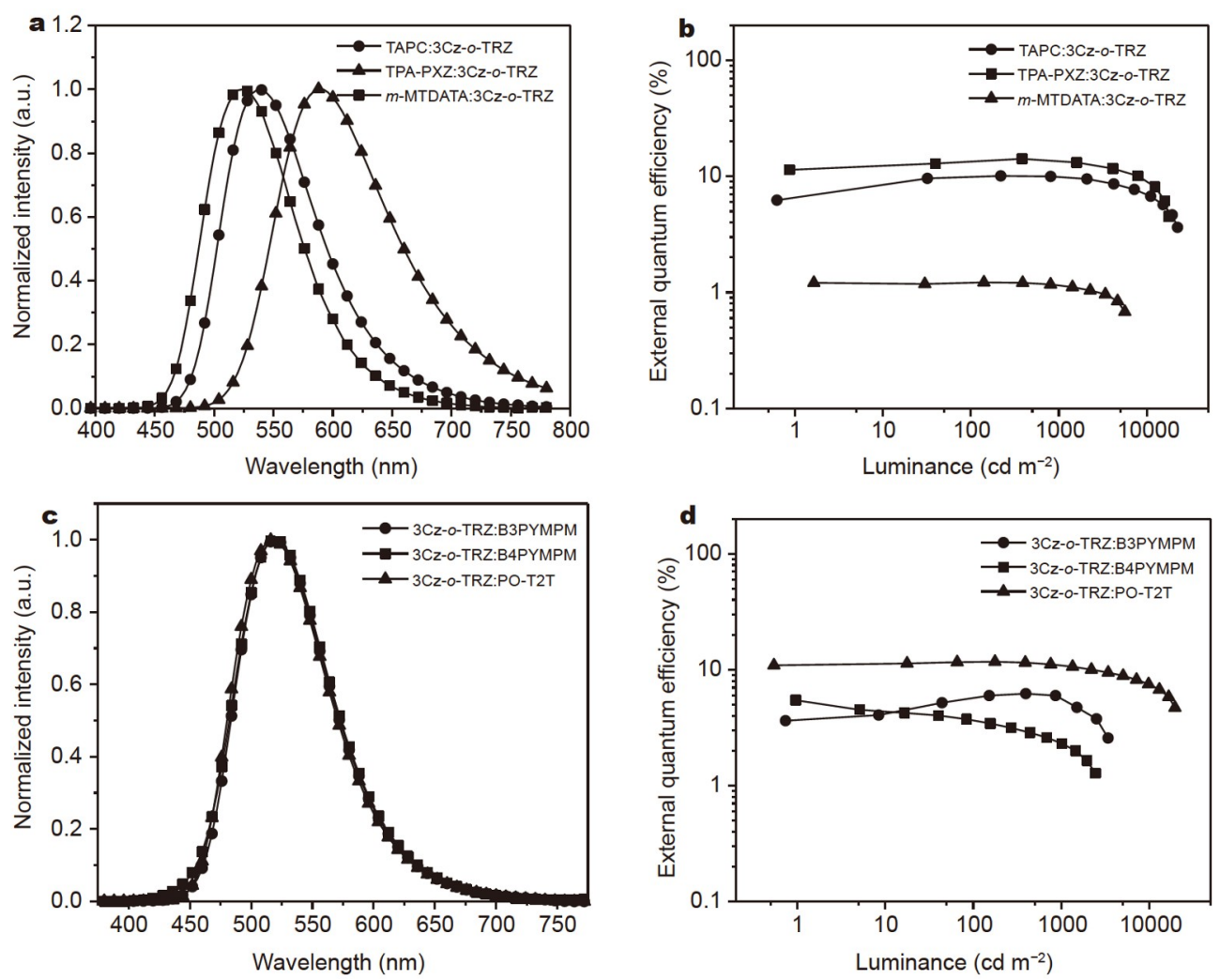

Figure 4 (a) EL spectra, (b) EQE-luminance plots of donors:3Cz-o-TRz; (c) EL spectra, (d) EQE-luminance plots of 3Cz-o-TRz:acceptors.

Table 1 Summary of OLED performances

\begin{tabular}{|c|c|c|c|c|c|c|}
\hline EML & $\lambda_{\max }(\mathrm{nm})$ & $V_{\text {on }}(\mathrm{V})$ & $\mathrm{CE}_{\max }\left(\mathrm{cd} \mathrm{A}^{-1}\right)$ & $\mathrm{EQE}_{\max }(\%)$ & $\mathrm{CE}_{1000}\left(\mathrm{~cd} \mathrm{~A}^{-1}\right)$ & $\mathrm{EQE}_{1000}(\%)$ \\
\hline $\begin{array}{c}\text { TAPC: } \\
\text { 3Cz-o-TRz }\end{array}$ & 520 & 2.7 & 39.3 & 12.1 & 37.4 & 11.5 \\
\hline $\begin{array}{l}\text { TPA-PXZ: } \\
\text { 3Cz-o-TRz }\end{array}$ & 540 & 2.6 & 33.8 & 10.1 & 33.0 & 9.9 \\
\hline $\begin{array}{c}\text { m-MTDATA: } \\
\text { 3Cz-o-TRz }\end{array}$ & 588 & 2.5 & 2.4 & 1.1 & 2.3 & 1.0 \\
\hline $\begin{array}{l}\text { 3Cz-o-TRz: } \\
\text { B3PyMPM }\end{array}$ & 516 & 2.6 & 16.0 & 5.5 & 7.0 & 2.3 \\
\hline $\begin{array}{l}\text { 3Cz-o-TRz: } \\
\text { B4PyMPM }\end{array}$ & 520 & 2.6 & 20.0 & 6.2 & 16.0 & 5.0 \\
\hline $\begin{array}{l}\text { 3Cz-o-TRz: } \\
\text { PO-T2T }\end{array}$ & 516 & 2.6 & 37.2 & 11.8 & 34.5 & 11.0 \\
\hline $\begin{array}{c}\text { TAPC:3Cz-o-TRz/3Cz- } \\
o \text {-TRz/3Cz-o-TRz:PO-T2T }\end{array}$ & 516 & 2.4 & 43.8 & 14.1 & 35.6 & 11.5 \\
\hline
\end{tabular}

$o$-TRz serving as either D or A component, the exciplex emitters could realize comparable EL performance with the maximum EQEs around $12 \%$. These results successfully demonstrate that our donor-spacer-acceptor structure molecule $3 \mathrm{Cz}-\mathrm{O}$-TRz meets the requirements of the multifunctional component to construct exciplexes and efficient exciplex emitters as either $\mathrm{D}$ or $\mathrm{A}$ component.

Given their similar green EL emission, TAPC:3Cz-o-TRz and 3Cz-o-TRz:PO-T2T were used to construct a simple tandem OLED to further demonstrate the superiority of $3 \mathrm{Cz}-0-\mathrm{TRz}$. The device configuration was ITO/TAPC $(30 \mathrm{~nm}) / \mathrm{TAPC}: 3 \mathrm{Cz}-\mathrm{o}-\mathrm{TRz}$
$(10 \mathrm{~nm}) / 3 \mathrm{Cz}-o-\mathrm{TRz} \quad(7 \mathrm{~nm}) / 3 \mathrm{Cz}-o-\mathrm{TRz}: \mathrm{PO}-\mathrm{T} 2 \mathrm{~T} \quad(10 \mathrm{~nm}) / \mathrm{PO}-$ T2T $(60 \mathrm{~nm}) / \mathrm{LiF}(1 \mathrm{~nm}) / \mathrm{Al}$. In this device, two individual emitters of TAPC:3Cz-o-TRz and 3Cz-o-TRz:PO-T2T were stacked in series through a thin $3 \mathrm{Cz}-\mathrm{o}-\mathrm{TRz}$ interconnecting layer. The interconnecting layer not only prevents the formation of an undesired interfacial exciplex between TAPC and PO-T2T [36] but also avoids additional charge injection barriers in the EML. The corresponding device performance is illustrated in Fig. 5. The EL spectrum of the tandem OLED was consistent with the emission of TAPC:3Cz-o-TRz and 3Cz-o-TRz:PO-T2T and exhibited no change with the increase in driving voltage. 

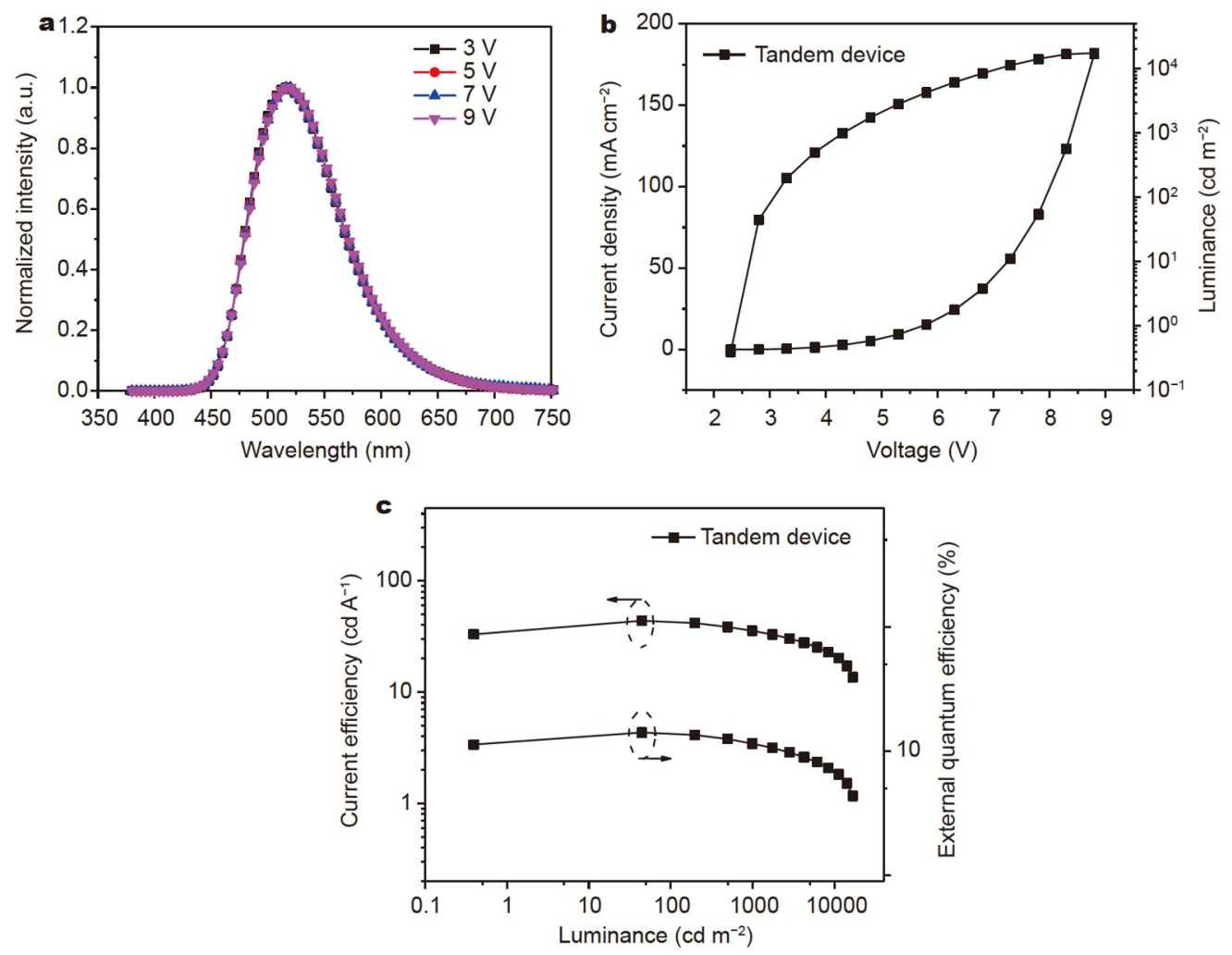

Figure 5 (a) EL spectra at different voltages; (b) current density-luminance-voltage characteristics, (c) CE-luminance plots, and EQE-luminance plots of the tandem device.

Accordingly, the tandem device originated from the emission of TAPC:3Cz-o-TRz and 3Cz-o-TRz:PO-T2T exciplexes. Compared with the OLEDs based on single exciplex emitters, the turn-on voltage of the tandem device further decreased to $2.4 \mathrm{~V}$ because the tandem OLED can successfully eliminate the charge injection barriers at the HTL/EML and ETL/EML interfaces, as shown in Fig. S9. Moreover, the series connection of two exciplex emitters could further avoid carrier leakage and improve the device efficiency $[37,38]$. Accordingly, the tandem OLED exhibited a maximum $\mathrm{CE}$ of $43.8 \mathrm{~cd} \mathrm{~A}^{-1}$ and a maximum EQE of $14.1 \%$, which improved by approximately $20 \%$ compared with those of TAPC:3Cz-o-TRz- and 3Cz-o-TRz:PO-T2T-based OLEDs. These results indicate that the appropriate design of device structure can evidently improve the EL performance of exciplex emitters.

\section{CONCLUSION}

A new donor-spacer-acceptor structure molecule, 3Cz-o-TRz, was developed using a diphenyl ether to connect the $3 \mathrm{Cz}$ and $\mathrm{TRz}$ segments. 3Cz-o-TRz can be used to construct exciplex emitters as either D or A component because it exhibits the intrinsic characteristics of $3 \mathrm{Cz}$ and $\mathrm{TRz}$ groups in the singlemolecule state. Moreover, TAPC:3Cz-o-TRz with $3 \mathrm{Cz}-\mathrm{o}$-TRz as the A component and 3Cz-o-TRz:PO-T2T with 3Cz-o-TRz as the $\mathrm{D}$ component achieved uniform EL performance with the maximum EQEs of $12.1 \%$ and $11.8 \%$, respectively. In addition, a simple tandem OLED formed by stacking TAPC:3Cz-o-TRz and 3Cz-o-TRz:PO-T2T in the EML realized improved performance with an ultralow turn-on voltage of $2.4 \mathrm{~V}$ and a maximum EQE of $14.1 \%$. These results prove that the donor-spacer-acceptor structure molecule not only serves as the multifunctional component to construct exciplex emitters but also has unique advantages in the design of device structures.

Received 30 April 2021; accepted 29 June 2021; published online 30 August 2021

1 Goushi K, Yoshida K, Sato K, et al. Organic light-emitting diodes employing efficient reverse intersystem crossing for triplet-to-singlet state conversion. Nat Photon, 2012, 6: 253-258

2 Goushi K, Adachi C. Efficient organic light-emitting diodes through up-conversion from triplet to singlet excited states of exciplexes. Appl Phys Lett, 2012, 101: 023306

3 Hung WY, Fang GC, Chang YC, et al. Highly efficient bilayer interface exciplex for yellow organic light-emitting diode. ACS Appl Mater Interfaces, 2013, 5: 6826-6831

4 Li J, Nomura H, Miyazaki H, et al. Highly efficient exciplex organic light-emitting diodes incorporating a heptazine derivative as an electron acceptor. Chem Commun, 2014, 50: 6174-6176

5 Liu XK, Chen Z, Zheng CJ, et al. Prediction and design of efficient exciplex emitters for high-efficiency, thermally activated delayedfluorescence organic light-emitting diodes. Adv Mater, 2015, 27: 23782383

6 Hung WY, Wang TC, Chiang PY, et al. Remote steric effect as a facile strategy for improving the efficiency of exciplex-based OLEDs. ACS Appl Mater Interfaces, 2017, 9: 7355-7361

7 Lin TC, Sarma M, Chen YT, et al. Probe exciplex structure of highly efficient thermally activated delayed fluorescence organic light emitting diodes. Nat Commun, 2018, 9: 3111

8 Al Amin NR, Kesavan KK, Biring S, et al. A comparative study via photophysical and electrical characterizations on interfacial and bulk exciplex-forming systems for efficient organic light-emitting diodes. ACS Appl Electron Mater, 2020, 2: 1011-1019 
9 Zhang M, Liu W, Zheng CJ, et al. Tricomponent exciplex emitter realizing over $20 \%$ external quantum efficiency in organic light-emitting diode with multiple reverse intersystem crossing channels. Adv Sci, 2019, 6: 1801938

10 Kim HB, Kim JJ. Recent progress on exciplex-emitting OLEDs. J Inf Display, 2019, 20: 105-121

11 Wang M, Huang YH, Lin KS, et al. Revealing the cooperative relationship between spin, energy, and polarization parameters toward developing high-efficiency exciplex light-emitting diodes. Adv Mater, 2019, 31: 1904114

12 Zhao J, Zheng C, Zhou Y, et al. Novel small-molecule electron donor for solution-processed ternary exciplex with $24 \%$ external quantum efficiency in organic light-emitting diode. Mater Horiz, 2019, 6: 14251432

13 Li M, Wang YF, Zhang DW, et al. Thermally activated delayed fluorescence material-sensitized helicene enantiomer-based OLEDs: A new strategy for improving the efficiency of circularly polarized electroluminescence. Sci China Mater, 2021, 64: 899-908

14 Lv X, Wang H, Meng L, et al. Highly efficient inverted organic lightemitting diodes based on thermally activated delayed fluorescence. Sci China Mater, 2016, 59: 421-426

15 Sun JW, Lee JH, Moon CK, et al. A fluorescent organic light-emitting diode with 30\% external quantum efficiency. Adv Mater, 2014, 26: 5684-5688

16 Jankus V, Chiang CJ, Dias F, et al. Deep blue exciplex organic lightemitting diodes with enhanced efficiency; P-type or E-type triplet conversion to singlet excitons? Adv Mater, 2013, 25: 1455-1459

17 Seino $\mathrm{Y}$, Inomata S, Sasabe H, et al. High-performance green OLEDs using thermally activated delayed fluorescence with a power efficiency of over $100 \mathrm{~lm} \mathrm{~W}^{-1}$. Adv Mater, 2016, 28: 2638-2643

18 Chen $\mathrm{D}$, Wang Z, Wang $\mathrm{D}$, et al. Efficient exciplex organic lightemitting diodes with a bipolar acceptor. Org Electron, 2015, 25: 79-84

19 Oh CS, Kang YJ, Jeon SK, et al. High efficiency exciplex emitters using donor-acceptor type acceptor material. J Phys Chem C, 2015, 119: 22618-22624

20 Zassowski P, Ledwon P, Kurowska A, et al. 1,3,5-Triazine and carbazole derivatives for OLED applications. Dyes Pigments, 2018, 149: 804-811

21 Liu W, Chen JX, Zheng CJ, et al. Novel strategy to develop exciplex emitters for high-performance OLEDs by employing thermally activated delayed fluorescence materials. Adv Funct Mater, 2016, 26: 20022008

22 Jeon SK, Yook KS, Lee JY. Highly efficient exciplex organic lightemitting diodes using thermally activated delayed fluorescent emitters as donor and acceptor materials. Nanotechnology, 2016, 27: 224001

23 Nguyen TB, Nakanotani H, Hatakeyama T, et al. The role of reverse intersystem crossing using a TADF-type acceptor molecule on the device stability of exciplex-based organic light-emitting diodes. Adv Mater, 2020, 32: 1906614

24 Siddiqui QT, Awasthi AA, Bhui P, et al. Thermally activated delayed fluorescence (green) in undoped film and exciplex emission (blue) in acridone-carbazole derivatives for OLEDs. J Phys Chem C, 2018, 123: 1003-1014

25 Jankus V, Data P, Graves D, et al. Highly efficient TADF OLEDs: How the emitter-host interaction controls both the excited state species and electrical properties of the devices to achieve near $100 \%$ triplet harvesting and high efficiency. Adv Funct Mater, 2014, 24: 6178-6186

26 Uoyama H, Goushi K, Shizu K, et al. Highly efficient organic lightemitting diodes from delayed fluorescence. Nature, 2012, 492: 234-238

27 Zhang Q, Li B, Huang S, et al. Efficient blue organic light-emitting diodes employing thermally activated delayed fluorescence. Nat Photon, 2014, 8: 326-332

28 Wang $\mathrm{K}$, Zheng CJ, Liu W, et al. Avoiding energy loss on TADF emitters: Controlling the dual conformations of D-A structure molecules based on the pseudoplanar segments. Adv Mater, 2017, 29: 1701476

29 Zeng W, Lai HY, Lee WK, et al. Achieving nearly 30\% external quantum efficiency for orange-red organic light emitting diodes by employing thermally activated delayed fluorescence emitters composed of 1,8-naphthalimide-acridine hybrids. Adv Mater, 2018, 30: 1704961

30 Zhang $\mathrm{T}$, Zhao B, Chu B, et al. Efficient exciplex emission from in tramolecular charge transfer material. Org Electron, 2015, 25: 6-11

31 Shi YZ, Wang K, Li X, et al. Intermolecular charge-transfer transition emitter showing thermally activated delayed fluorescence for efficient non-doped OLEDs. Angew Chem Int Ed, 2018, 57: 9480-9484

32 Yang HY, Zheng CJ, Zhang M, et al. Green solution-processed thermally activated delayed fluorescence OLEDs with improved performance by using interfacial exciplex host. Org Electron, 2019, 73: 36-42

33 Hirata S, Sakai Y, Masui K, et al. Highly efficient blue electroluminescence based on thermally activated delayed fluorescence. Nat Mater, 2015, 14: 330-336

34 Zeng X, Huang YH, Gong S, et al. Rational design of perfectly oriented thermally activated delayed fluorescence emitter for efficient red electroluminescence. Sci China Mater, 2021, 64: 920-930

35 Zhang M, Wang K, Zheng CJ, et al. Development of red exciplex for efficient OLEDs by employing a phosphor as a component. Front Chem, 2019, 7: 16

36 Zhao B, Zhang H, Miao Y, et al. High color stability and CRI $(>80)$ fluorescent white organic light-emitting diode based pure emission of exciplexes by employing merely complementary colors. J Mater Chem C, 2018, 6: 304-311

37 Qiao X, Tao Y, Wang Q, et al. Controlling charge balance and exciton recombination by bipolar host in single-layer organic light-emitting diodes. J Appl Phys, 2010, 108: 034508

38 Reineke S, Schwartz G, Walzer K, et al. Reduced efficiency roll-off in phosphorescent organic light emitting diodes by suppression of triplettriplet annihilation. Appl Phys Lett, 2007, 91: 123508

Acknowledgements This work was supported by the National Natural Science Foundation of China (51773029, 52073040, 51533005 and 51821002), the Fundamental Research Funds for the Central Universities (ZYGX2016Z010), and the International Cooperation and Exchange Project of Science and Technology Department of Sichuan Province (2019YFH0057 and 2019YFH0059).

Author contributions Zheng CJ and Zhang XH conceived and supervised the project. Yang HY and Shi YZ designed and synthesized the molecule. Yang HY, Zhang M, and Zhao JW prepared the device and performed the corresponding measurements and characterizations. Yang HY and Pu CP performed the data analysis. Yang HY wrote the paper with support from Zheng CJ. All authors contributed to the general discussion.

Conflict of interest The authors declare that they have no conflict of interest.

Supplementary information Experimental details and supporting data are available in the online version of the paper.

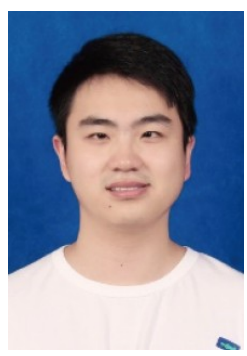

Hao-Yu Yang is a PhD student of the Organic Optoelectronic Laboratory at the University of Electronic Science and Technology of China. His research focuses on the organic optoelectronic materials and devices, mainly on the design, synthesis, manufacture, and characterization of thermally activated delayed fluorescent and exciplex materials for OLEDs. 


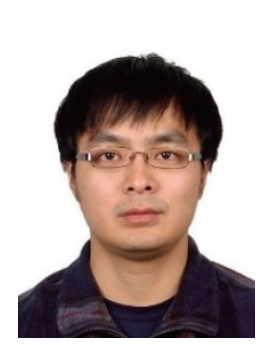

Cai-Jun Zheng was born in 1984. He received his BSc degree from the Department of Chemistry, Tsinghua University in 2005, and $\mathrm{PhD}$ degree from Technical Institute of Physics and Chemistry, Chinese Academy of Sciences in 2010. He joined the University of Electronic Science and Technology of China as a professor in 2016. Currently his interest mainly focuses on the organic optoelectronic materials and devices.

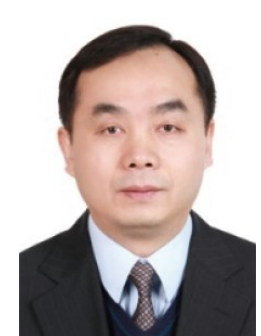

Xiao-Hong Zhang was born in 1967. He received his $\mathrm{BSc}$ and MSc degrees from the Department of Materials Science and Engineering, Beijing University of Aeronautics and Astronautics in 1989 and 1992, and $\mathrm{PhD}$ degree from the Department of Chemical Engineering and Materials Science, Beijing University of Technology in 1996. He joined Soochow University as a professor in 2013. His current interests mainly focus on organic optoelectronic materials and devices and semiconductor nano-materials and devices.
一种新型给体-间隔基-受体结构材料作为多功能组 分实现高效激基复合物发光二极管

杨浩宇 ${ }^{1,2}$, 郑才俊 ${ }^{*}$, 张明 ${ }^{1,2}$, 赵决文 ${ }^{1}$, 史益忠 ${ }^{2}$, 蒲春鹏 ${ }^{1}$, 林慧 ${ }^{1}$, 陶斯禄 ${ }^{1}$, 张晓宏 $^{2^{*}}$

摘要 为了实现高效的激基复合物发光二极管(OLED), 我们设计并合 成了一种新型给体-间隔基-受体 (D-Spacer-A)结构分子 $3 \mathrm{Cz}-o$-TRz. 通 过引入二苯醚基团作为间隔基, 可以有效地抑制 $3 \mathrm{Cz}-\mathrm{o}-\mathrm{TRz}$ 分子内的电 荷转移过程, 使其在单分子态下能够同时表现出给体和受体片段的本 征特性, 从而能够作为多功能组分来构建激基复合物. 基于此, $3 \mathrm{Cz}-\mathrm{o}$ $\mathrm{TRz}$ 分别与不同的受体材料和给体材料结合, 构建了六种激基复合物发 光分子. 其中, 基于 $3 \mathrm{Cz}-o-\mathrm{TRz}$ 分别作为给体和受体组分所构建的激基 复合物 OLED器件均实现了最大外部量子效率(EQE)约为 $12 \%$ 的性能. 并且, 通过串联激基复合物 TAPC:3Cz-o-TRz和 $3 \mathrm{Cz}-o-\mathrm{TRz}: \mathrm{PO}-\mathrm{T} 2 \mathrm{~T}$ 为发 光分子进一步提高了器件性能, 最大EQE可达 $14.1 \%$. 我们的工作证明 了具有 D-Spacer-A结构的分子在构建激基复合物OLED中具有巨大潜 力. 